\title{
Current treatment options for advanced choriocarcinoma on the basis of own case and review of the literature
}

\author{
Patrycja Wreczycka-Cegielny ${ }^{1,2}$, Tomasz Cegielny ${ }^{2}$, Marcin Oplawski ${ }^{1}$, \\ Wlodzimierz Sawicki ${ }^{3}$, Zbigniew Kojs ${ }^{4}$ \\ 'Gyneacology and Obstetrics Ward with a Section of Oncological Gynaecology, \\ Ludwik Rydygier Specialist Hospital in Cracow, Poland \\ ${ }^{2}$ Medical Didactics Department, Faculty of Medicine, Jagiellonian University, Cracow, Poland \\ ${ }^{3}$ The Chair and Clinic of Obstetrics, Gynaecologic Diseases and Oncological Gynaecology of the Second Faculty \\ of Medicine of Warsaw Medical University, Poland \\ ${ }^{4}$ Oncological Gynaecology Chair, Oncology Centre — Maria Skłodowska-Curie Institute, Cracow Branch, Poland
}

\begin{abstract}
Choriocarcinoma is one of rare neoplasms of female reproductive organs. In the last decade only a few cases of this disease have been registered in Poland. The paper presents the current principles of diagnosis and treatment options for this rare disease based on the authors' clinical experience (description of the case) and literature review. This paper provides basic information on epidemiology, FIGO classification, and also discusses the chemotherapy regimens used in the medical treatment of choriocarcinoma. Surgical options were also considered.
\end{abstract}

A thematic review of the most important papers published in international journals in recent years has also been made. Key words: choriocarcinoma; trophoblast tumour; gestational trophoblastic disease

Ginekologia Polska 2018; 89, 12: 711-715

\section{INTRODUCTION}

Choriocarcinoma belongs to a group of rare gynaecological cancers, being one of the most aggressive forms of gestational trophoblastic disease. An increased risk of developing choriocarcinoma is observed in the cases of early or late pregnancy $(<19,>35$ years of age), in particular, among women above 40 years of age, in whose case the risk of molar pregnancy (hydatidiform mole) is then approximately 7.5-fold higher [1-3]. Complete molar pregnancy in the pregnancy which preceded the diagnosis of the gestational cancer, increase the risk of malignant trophoblastic tumours almost 1000-fold and seems to be the most significant prognostic factor. Molar pregnancy may reoccur in about $1 \%$ patients [2]. The risk of transformation into choriocarcinoma in the case of complete molar pregnancy is estimated to be $2-3 \%$, whilst in the case of partial molar pregnancy it is regarded to equal $<0.5 \%[4,5]$. Gestational choriocarcinoma arising in the fallopian tube or corneal ectopic pregnancyhas been reported [6-8]. The prevalence of choriocarcinoma in Europe and North America is evaluated to be $1 / 40,000$ pregnancies and in $1 / 40$ cases of molar pregnancy. The risk of developing this condition in women from South-East Asia and Japan is higher and totals 9.2 and $3.3 / 40000$ pregnancies respectively [1,9]. In Poland, an epidemiological analysis is difficult, given the casuistry of the problem. In 2002-2012, 65 cases of malignant placental tumours were reported (code: C58 according to ICD10) and they accounted for the deaths of 7 women [10].

Patients with choriocarcinoma may sometimes complain about abnormal vaginal bleeding. In trophoblast-originating tumours, the metastatic foci are formed via blood circulatory spread in the lungs (80\%), vagina $(30 \%)$, brain (10\%) and liver (10\%). The lung metastases are usually asymptomatic, but they may also cause dyspnoea, cough, 
haemoptysis and chest pain. The vaginal metastases are the cause of bleeding, often with an intensity difficult to control due to the rich pathological tumour vascularisation [1].

According to the anatomical classification (FIGO 2002), the disease in the first stage is limited solely to the uterus; in the second - the lesion exceeds beyond the uterus, but is limited to the reproductive organ; in the third one - the metastases are found in the lungs irrespectively of the involvement of the reproductive organ; in the fourth one — the metastases are spread to other organs [11].

The risk factors (evaluated respectively as $0,1,2,4$ points) in the above classification, comprise age $(<40,>40$ years), the manner of ending the pregnancy (molar pregnancy, miscarriage natural timely delivery), the time span from the end of the pregnancy $(<4,4-7,7-12,>12$ months), $\beta$-hCG level $\left(<10^{3}\right.$, up to $10^{4}$, up to $\left.10^{5},>10^{5}\right)$, tumour size $(0.3-5,>5 \mathrm{~cm})$, the presence of distant metastases (lungs, spleen/kidneys, alimentary tract, brain/liver), the number of metastases $(0,1-4,5-8,>8)$ and previous chemotherapy (monochemotherapy/polychemotherapy). On the basis of this classification, when the score is below 6 points, the risk of monochemotherapy resistance is defined as low, whilst above this score - as high. The choice of therapeutic regimen is the consequence of the analysis of the above criteria.

The treatment of choriocarcinoma is based first of all on chemotherapy, yet surgery and radiotherapy are also sometimes included. Hysterectomy in the low-risk group patients may shorten the time of the necessary chemotherapy, and this is beneficial, especially in women who have decided to discontinue reproduction. In the patients in the high risk group (with metastases), hysterectomy does not improve the treatment outcomes and therefore is not recommended [12]. Intervention surgery plays an important role, as massive haemorrhages from the highly vascularised tumour are likely to happen. The scope of surgical procedures may be varied. Usually it is hysterectomy, but one must not forget about possible bleeding from the metastases located in the lungs or brain. Neurosurgical treatment is applied also in order to decrease intracranial pressure [13]. Sometimes it is advisable to resect the persistent metastatic foci in parenchymal organs, in particular when these are isolated foci.

Radiotherapy is applied in the cases of brain metastases, most frequently in a form of whole brain irradiation, often with intrathecal administration of methotrexate [14]. Multidrug chemotherapy without radiotherapy can be an alternative for this subgroup of patients. Gavanie et al. retrospectively analyzed 21 patients with brain metastases from gestational trophoblastic neoplasia treated by this way and showed high survival rate [15]. There is no consensus on optimal treatment of brain metastases [16]. Radiotherapy may also be administered in the area of minor pelvis in order to treat the residua focus of the tumour [17] or a non-resectable tumour causing recurrent bleeding [18].

Below we discuss the treatment options in a case of a 35-year old woman three months after the third childbirth (vaginal delivery) with an intensive bleeding from the reproductive organ and some cough which had been intensifed for a few days. The last pregnancy and childbirth - similarly as the previous ones - were uneventful. In the gynaecological examination a tumour was found in the minor pelvis $(8 \mathrm{~cm}$ in diameter) as well as some brittle infiltration, bleeding upon contact, filling almost the entire vaginal lumen, which, upon histopathological assessment turned out to be choriocarcinoma. The abdominal and pelvic CT scan revealed an enlarged uterine body with polycyclic contour and fluid space inside the uterine cavity; the vaginal walls were infiltrated with tumour, whilst no pathologies were found in the remaining organs under examination. Head CT did not reveal any abnormalities, yet in the chest numerous metastases were found. The baseline of $\beta$-human chorionic gonadotopin ( $\beta$-hCG) level equaled $52776.02 \mathrm{U} / \mathrm{L}$. With respect to the diagnosis of choriocarcinoma with lung metastases, and upon risk stratification (score: 9 points), the patient was qualified to chemotherapy with the EMA-CO regimen. After the administration of 1 course of chemotherapy, the patient's condition was significantly deteriorated - she was referred to an intensive care unit in a serious condition and unconscious. At that time the findings comprised thrombocytopenia, neutropenia and anaemia. After the transfusion of packed red blodd cells (PRBCs) and the administration of granulocyte colony-stimulating factor (GCSF) and leucovorin, as well as supportive treatment, a significant improvement of the patient's general condition was obtained together with a decrease of $\beta$-hCG concentration to the level of $4041.0 \mathrm{U} / \mathrm{L}$. After 14 days of the stabilisation of the patient a decision was taken to resume chemotherapy. The patient received three courses of chemotherapy and $\beta$-hCG level dropped down to $88.82 \mathrm{U} / \mathrm{L}$. Cancer infiltration in the vagina was significantly reduced. In spite of the above, the patient suffered from a vaginal bleeding (the loss of approx. $2000 \mathrm{~mL}$ ). With respect to the life-threatening condition the patient was qualified for an urgent laparotomy. During the intervention bleeding to the peritoneal cavity from an infiltrate in the uterine wall was found, so the internal iliac arteries were ligated and then hysterectomy was performed. Full haemostasis was achieved in the pelvis and bleeding from an infiltrate in the vagina was brought under control. In post-operative course, 8 units of PRBCs and 5 units of fresh frozen plasma (FFP) were transfused.

The histopathological assessment of the post-operative specimen revealed extensive necrotic areas in the body of the uterus which exceeded the thickness of the uterine muscle and also some cancer foci in the uterine cervix and 
adnexa and in some fragments of the vaginal wall. After the next two weeks from the surgery, chemotherapy was possible to be resumed. However, another bleeding episode from the flat vaginal infiltration occurred, therefore high dose rate (HDR) brachytherapy was carried out at the dose of $21 \mathrm{~Gy}$ in three fractions, after which the residua infiltrate completely regressed. After four courses of chemotherapy, partial regression of the lung lesions was obtained and $\beta$-HCG level was stabilized, yet without normalization. The treatment so far was regarded as potentially efficacious and therefore it was continued according to the EMAICO regimen.

During the ninth course of therapy the $\beta$-hCG level elevation was observed and therefore the regimen was changed into EMA EP. After two series the marker level was normalised (2.31 U/L), yet during the next course, another episode of $\beta$-hCG elevation was observed (17.32 U/L). Chemotherapy: paclitaxel + cisplatin + etoposide was then implemented. After two courses, the PET-CT examination was performed and the result suggested the presence of active neoplasia in the vaginal stump and two active cancer foci in both lungs. The specimens collected from the vaginal stump revealed choriocarcinoma in pathomorphological assessment. Teleradiotherapy of the minor pelvic area was performed with a dose of $50 \mathrm{~Gy}$ in 25 fractions. After the end of irradiation, given an elevated level of $\beta$-hCG ( $62 \mathrm{U} / \mathrm{L}, 203 \mathrm{U} / \mathrm{L}$ ) the treatment with paclitaxel + cisplatin was implemented with a good tolerance. The follow-up assessment revealed persistent metastases in the lungs. With regards to the young age of the patient and a good general condition, metastasectomy was performed at the Thoracic Surgery Clinic of the Collegium Medicum of the Jagiellonian University in Cracow was performed. The histopathological assessment confirmed the type of the tumours (choriocarcinoma). Within the further chemotherapy course, a significant progression of the $\beta$-hCG marker occurred: $13057 \mathrm{U} / \mathrm{L}$. With regards the aggressive course of the disease, the treatment was modified. An attempt was made to treat the patient according to the regimen with cyclophosphamide + methotrexate. The patient did not turn out for the continuation of the treatment.

\section{DISCUSSION AND REVIEW OF THE LITERATURE}

As it is seen from the published data and from the treatment algorithms applicable, the treatment of choice in the therapy of choriocarcinoma is chemotherapy, yet surgical treatment may be necessary, especially in the cases of life-threatening complications, most frequently connected with a massive bleeding from the tumour.

More than $90 \%$ patients respond to systemic therapy. The prognosis is better in the cases when the disease is limited only to the uterus or when metastases are present in other locations than liver and brain, when the period from the last pregnancy is shorter and the baseline level of $\beta$-hCG upon qualification for treatment was lower. The treatment consists in chemotherapy with methotrexate or actinomycin $D$ in mono-therapy or multi-drug regimens (e.g. EMAICO with additional use of etoposide, cyclophosphamide and vincristine). An indication for chemotherapy is such pattern of choriocarcinoma, in which chemotherapy is applied until the normalisation of $\beta$-hCG level plus two additional cycles. In the case of metastases found in the patients from low risk group, monotherapy is used, whereas in the case of resistance or high risk - multi-drug regimen (EMAICO). In some cases of a high-risk disease, even 3-4 additional chemotherapy cycles are recommended in order to minimise the risk of recurrence [19]. In the follow-up after the treatment of choriocarcinoma, the level of $\beta$-hCG must be established every 4 weeks up to 6 months post treatment, then every 2 months, and after 12 months up to 5 years after treatment - every 6 months. The proposed follow-up patterns after choriocarcinoma treatment differ from each other; some practitioners suggest even the measurement of $\beta$-hCG level every 6 months till end of the patient's life [19].

In 2016 Lawrie et al., presented a systematic overview made on the basis of the Cochrane database: comprising seven randomised clinical trials $(\mathrm{RCT})$ describing the treatment of more than 600 patients with choriocarcinoma, in which the effectiveness and toxicity of methotrexate and actinomycin $D$ administered in monotherapy were compared. The efficacy of the treatments was comparable and no significant differences concerning the side effects were found (nausea, vomiting, diarrhoea, anaemia, neutropenia, thrombocytopenia); only in the case of methotrexate, some lower rate of alopecia was observed. In the opinion of the authors, the use of actinomycin $D$ probably allows for a faster primary healing in a low-risk group of women, whilst the rate of treatment failures seems to be lower than in the case of therapy based on methotrexate [20]. Three years earlier, another overview of the research studies evaluating the efficacy of the treatment of high-risk trophoblastic cancers (high-risk gestational trophoblastic neoplasms (GTN), > 6 points in FIGO) was made also on the basis of the Cochrane. The authors analysed and compared the CHAMOCA, MAC and EMA/CO regimens. It was found that the CHAMOCA multi-drug therapy (cyclophosphamide, hydroxy-urea, actinomycin D, methotrexate, doxorubicin, melphalan and vincristine) is not recommended for the treatment of malignant trophoblastic tumours, as it is more toxic and less efficient than the MAC regimen (methotrexate, actinomycin $D$, chlorambucil). Chemotherapy with EMA/CO regime is currently the most frequently used first line therapy in the case of high risk patients, although this regimen was not compared with other drug combinations, such as 
MAC, in (RCT). The authors would like to note that some further evaluation of the above regimens is recommended in well designed RCTs, yet with regards to a low number of such diagnoses, the trials in this area are very difficult and thus a multi-centre collaboration is necessary [21]. Complete remission with oral etoposide without any significant toxicity for refractory choriocarcinoma has been reported [22].

Last year Cochrane Database published another systematic overview, this time evaluating the role of prophylactic chemotherapy applied in women with diagnosed mole pregnancy, as a preventive measure against the development of trophoblastic cancers. The analysis of almost 90 papers allowed to conclude that a prophylactic chemotherapy may decrease the risk of progression to GTN in women with complete mole pregnancy, in whose case there is a high risk of malignant transformation, yet the current evidence for the benefits of this therapy is quite limited by their low quality and a small number of patients in the analysed trials. Preventive chemotherapy may increase drug resistance, delay healing and cause side effects and that is why it cannot be routinely recommended and used [23]. Braga et al. evaluated expectant versus immediate chemotherapy following pathological diagnosis of gestational choriocarcinoma in patients with nonmetastatic disease and concluded that close surveillance of women with pathological diagnosis of nonmetastatic gestational choriocarcinoma seems to be a safe practice [24].

\section{SUMMARY}

Our experience based among others of the presented case and world recommendations show that synchronous lung metastases developing three months after childbirth prove a very dynamic character of this carcinoma. The presence of such metastases qualified our patient discussed here to the group with the third stage of the disease (FIGO classification) and the risk factors analysis conducted in the above scale (high risk patient) provided grounds for an inclusion of the EMA/CO polychemotherapy. This regimen is effective in $80 \%$ patients who were not systemically treated before.

The therapy of the patients with choriocarcinoma is usually long, often it is necessary to apply a dozen chemotherapy cycles, which leads to an accumulation of side effects and in the majority of patients it is also necessary to introduce supportive therapy. As many as even 1/3 high risk patients develop chemo-resistance. In such a situation the regimen must be modified or some other treatment methods need to be introduced, metastasectomy or radiotherapy. The decision to resume oncological treatment after the surgery or to modify chemotherapy regimen or to apply radiotherapy should be taken by an experienced oncogynaecologist in consultation with an interdisciplinary cancer board. The patients with such a rare diagnosis should be treated in highly specialised centres and by experienced specialists sothat a vast range of individualised treatment could be possible.

In spite of numerous chemotherapy regimens or the implementation of surgical treatment and radiotherapy, a complete therapeutic success is still not possible in every case in about $10-25 \%$ cases of 2 and 3 stage patients as defined by FIGO. The causes for the treatment failures in our patient, occurring in spite of the maximum efforts to treat her and the use of numerous methods can be found in the simultaneous presence of many adverse circumstances such as advancement stage, chemo-resistance and life-threatening complications. Moreover, about $70 \%$ patients in whose case treatment was not successful, are the women in whom choriocarcinoma developed after the childbirth - this condition was also present in the above case [5].

\section{Conflict of interests}

The authors do not report any conflict of interests.

\section{The authors' contributions}

PW-C: the concept and objectives of the paper, preparation of the draft, TC: literature overview and draft preparation, MO: draft preparation, ZK: co-author of the concept of the paper, draft correction, WS: draft review. All the authors read and accepted the final version of the paper.

\section{REFERENCES}

1. Lurain JR. Gestational trophoblastic disease I: epidemiology, pathology, clinical presentation and diagnosis of gestational trophoblastic disease, and management of hydatidiform mole. Am J Obstet Gynecol. 2010; 203(6): 531-539, doi: 10.1016/j.ajog.2010.06.073, indexed in Pubmed: 20728069.

2. Goldstein DP, Berkowitz RS. Current management of gestational trophoblastic neoplasia. Hematol Oncol Clin North Am. 2012; 26(1): 111-131, doi: 10.1016/j.hoc.2011.10.007, indexed in Pubmed: 22244665.

3. Tarney CM, Tian C, Craig ER, et al. Relative Effects of Age, Race, and Stage on Mortality in Gestational Choriocarcinoma. Int J Gynecol Cancer. 2018; 28(2): 338-345, doi: 10.1097/IGC.0000000000001156, indexed in Pubmed: 29232272

4. Soper JT. Gestational trophoblastic disease. Obstet Gynecol. 2006; 108(1): 176-187, doi: 10.1097/01.AOG.0000224697.31138.a1, indexed in Pubmed: 16816073.

5. Seckl M, Sebire N, Berkowitz R. Gestational trophoblastic disease. The Lancet. 2010:376(9742):717-729, doi: 10.1016/s0140-6736(10)60280-2.

6. Lin LH, Fushida K, Hase EA, et al. Gestational Tubal Choriocarcinoma Presenting as a Pregnancy of Unknown Location following Ovarian Induction. Case Rep Obstet Gynecol. 2018; 2018: 4705192, doi: 10.1155/2018/4705192, indexed in Pubmed: 29854512.

7. Han V, Kaye S. A Rare Case of Gestational Choriocarcinoma Presenting as Cornual Ectopic Pregnancy. J Obstet Gynaecol Can. 2018;40(3): 351-353, doi: 10.1016/j.jogc.2017.08.009, indexed in Pubmed: 29032067.

8. Liu S, Fan C, Feng F, et al. Prognosis of gestational choriocarcinoma diagnosed incidentally during laparoscopy for a presumed cornual pregnancy: a report of five cases. Cancer Biol Med. 2017; 14(2): 191-193, doi: 10.20892/j.issn.2095-3941.2017.0004, indexed in Pubmed: 28607811.

9. $\mathrm{Ngan} \mathrm{H}$, Bender H, Benedet JL, et al. Gestational trophoblastic neoplasia, FIGO 2000 staging and classification. International Journal of Gynecology \& Obstetrics. 2004; 83: 175-177, doi: 10.1016/s0020-7292(03)90120-2.

10. Wojciechowska U, Didkowska J. Zachorowania i zgony na nowotwory złośliwe w Polsce. KRN, Centrum Onkologii, Instytut Marie SkłodowskiejCurie, www.onkologia.org.pl. 2014. 
11. FIGO Oncology Committee. FIGO staging for gestational trophoblastic neoplasia 2000. FIGO Oncology Committee. Int J Gynaecol Obstet. 2002; 77(3): 285-287, indexed in Pubmed: 12065144.

12. Suzuka K. Adjuvant hysterectomy in low-risk gestational trophoblastic disease. Obstetrics \& Gynecology. 2001; 97(3): 431-434, doi: 10.1016/s0029-7844(00)01169-8.

13. Mangili G, Lorusso D, Brown J, et al. Trophoblastic disease review for diagnosis and management: a joint report from the International Society for the Study of Trophoblastic Disease, European Organisation for the Treatment of Trophoblastic Disease, and the Gynecologic Cancer InterGroup. Int J Gynecol Cancer. 2014; 24(9 Suppl 3): S109-S116, doi: 10.1097/IGC.0000000000000294, indexed in Pubmed: 25341573.

14. Soper JT. Role of surgery and radiation therapy in the management of gestational trophoblastic disease. Best Pract Res Clin Obstet Gynaecol. 2003; 17(6): 943-957, indexed in Pubmed: 14614891.

15. Gavanier D, Leport H, Massardier J, et al. Gestational trophoblastic neoplasia with brain metastasis at initial presentation: a retrospective study. Int J Clin Oncol. 2018 [Epub ahead of print], doi: 10.1007/s10147018-1337-9, indexed in Pubmed: 30242539.

16. Frost AS, Sherman JH, Rezaei K, et al. Choriocarcinoma with brain, lung and vaginal metastases successfully treated without brain radiation or intrathecal chemotherapy: A case report. Gynecol Oncol Rep. 2017; 20: 97-99, doi: 10.1016/j.gore.2017.03.014, indexed in Pubmed: 28393093.

17. Hanna RK, Soper JT. The role of surgery and radiation therapy in the management of gestational trophoblastic disease. Oncologist. 2010;
15(6): 593-600, doi: 10.1634/theoncologist.2010-0065, indexed in Pubmed: 20495216.

18. Vaid N, Kaur C, Vidya S, et al. A Case Report on Radiotherapy in Choriocarcinoma: A Life Saving Procedure. Asia-Oceania Journal of Obstetrics and Gynaecology. 2010; 20(3): 245-247, doi: 10.1111/j.1447-0756.1994. tb00464.x.

19. Gestational Trophoblastic Disease. 2012, doi: 10.1007/978-1-61779394-3.

20. Lawrie T, Alazzam M, Tidy J, et al. First-line chemotherapy in low-risk gestational trophoblastic neoplasia. Cochrane Database of Systematic Reviews. 2016, doi: 10.1002/14651858.cd007102.pub4.

21. Deng L, Zhang J,WuT, et al. Combination chemotherapy for primary treatment of high-risk gestational trophoblastic tumour. Cochrane Database of Systematic Reviews. 2013, doi: 10.1002/14651858.cd005196.pub4.

22. Dhanushkodi M, Ganesan T, Sagar TG. Refractory Choriocarcinoma: Complete Response With Oral Etoposide. J Glob Oncol. 2017; 3(5): 678-679, doi: 10.1200/JGO.2016.006049, indexed in Pubmed: 29094106.

23. Wang Q, Fu J, Hu L, et al. Prophylactic chemotherapy for hydatidiform mole to prevent gestational trophoblastic neoplasia. Cochrane Database of Systematic Reviews. 2017, doi: 10.1002/14651858.cd007289.pub3.

24. Braga A, Campos V, Filho JR, et al. Is chemotherapy always necessary for patients with nonmetastatic gestational trophoblastic neoplasia with histopathological diagnosis of choriocarcinoma? Gynecol Oncol. 2018; 148(2): 239-246, doi: 10.1016/j.ygyno.2017.12.007, indexed in Pubmed: 29248195. 